\title{
Comparative proteome analysis reveals conserved and specific adaptation patterns of Staphylococcus aureus after internalization by different types of human non-professional phagocytic host cells
}

\author{
Kristin Surmann ${ }^{1}$, Stephan Michalik ${ }^{2}$, Petra Hildebrandt ${ }^{2}$, Philipp Gierok ${ }^{3}$, Maren Depke ${ }^{2}$, \\ Lars Brinkmann ${ }^{1}$, Jörg Bernhardt ${ }^{4}$, Manuela G. Salazar ${ }^{1}$, Zhi Sun ${ }^{5}$, David Shteynberg ${ }^{5}$, \\ Ulrike Kusebauch ${ }^{5}$, Robert L. Moritz ${ }^{5}$, Bernd Wollscheid ${ }^{6}$, Michael Lalk ${ }^{3}$, Uwe Völker ${ }^{1}$ and \\ Frank Schmidt ${ }^{2 *}$ \\ 1 Interfaculty Institute for Genetics and Functional Genomics, University Medicine Greifswald, Greifswald, Germany \\ 2 ZIK-FunGene Junior Research Group Applied Proteomics, Interfaculty Institute for Genetics and Functional Genomics, University Medicine Greifswald, \\ Greifswald, Germany \\ ${ }^{3}$ Institute of Biochemistry, Ernst-Moritz-Arndt-University Greifswald, Greifswald, Germany \\ ${ }^{4}$ Institute for Microbiology, Ernst-Moritz-Arndt-University Greifswald, Greifswald, Germany \\ ${ }^{5}$ Institute for Systems Biology, Seattle, WA USA \\ ${ }^{6}$ Institute of Molecular Systems Biology, ETH Zurich, Zurich, Switzerland
}

\section{Edited by:}

Susann Müller, Helmholtz Centre for Environmental Research Leipzig Germany, Germany

Reviewed by:

Patrick K. H. Lee, City University of Hong Kong, Hong Kong

Nico Jehmlich, Helmholtz-Centre for Environmental Research - UFZ,

Germany

*Correspondence:

Frank Schmidt, Junior Research

Group Applied Proteomics, Interfaculty Institute for Genetics and Functional Genomics,

ZIK-FunGene University Medicine

Greifswald

Friedrich-Ludwig-Jahn-Str. 15a, 17475 Greifswald, Germany e-mail: frank.schmidt@unigreifswald.de
Staphylococcus aureus is a human pathogen that can cause a wide range of diseases. Although formerly regarded as extracellular pathogen, it has been shown that $S$. aureus can also be internalized by host cells and persist within these cells. In the present study, we comparatively analyzed survival and physiological adaptation of S. aureus HG001 after internalization by two human lung epithelial cell lines (S9 and A549), and human embryonic kidney cells (HEK 293). Combining enrichment of bacteria from host-pathogen assays by cell sorting and quantitation of the pathogen's proteome by mass spectrometry we characterized $S$. aureus adaptation during the initial phase between $2.5 \mathrm{~h}$ and $6.5 \mathrm{~h}$ post-infection. Starting with about $2 \times 10^{6}$ bacteria, roughly $1450 \mathrm{~S}$. aureus proteins, including virulence factors and metabolic enzymes were identified by spectral comparison and classical database searches. Most of the bacterial adaptation reactions, such as decreased levels of ribosomal proteins and metabolic enzymes or increased amounts of proteins involved in arginine and lysine biosynthesis, enzymes coding for terminal oxidases and stress responsive proteins or activation of the sigma factor SigB were observed after internalization into any of the three cell lines studied. However, differences were noted in central carbon metabolism including regulation of fermentation and threonine degradation. Since these differences coincided with different intracellular growth behavior, complementary profiling of the metabolome of the different non-infected host cell types was performed. This revealed similar levels of intracellular glucose but host cell specific differences in the amounts of amino acids such as glycine, threonine or glutamate. With this comparative study we provide an impression of the common and specific features of the adaptation of $S$. aureus HG001 to specific host cell environments as a starting point for follow-up studies with different strain isolates and regulatory mutants.

Keywords: Staphylococcus aureus, human cell lines, host-pathogen interaction, proteomics, label-free quantitation

\section{INTRODUCTION}

The Gram-positive bacterium Staphylococcus aureus is known to be a commensal and colonizes $20-30 \%$ of the human population without causing any symptoms. However, it is also the cause of many infectious diseases in humans ranging from mild skin infections to toxin-mediated diseases like the toxic shock syndrome as well as endocarditis, pneumonia, and septicaemia (Lowy, 1998; Wertheim et al., 2005). Though formerly regarded to be an extracellular pathogen it has also been shown that $S$. aureus is able to invade, persist and replicate inside non-professional phagocytotic cells (Hudson et al., 1995; Almeida et al., 1996; Garzoni and Kelley, 2009; Tuchscherr et al., 2011). Furthermore, S. aureus infects different types of organs and cells (Wertheim et al., 2005; Haslinger-Löffler et al., 2006).

Adaptation of $S$. aureus to the intracellular environment of host cells likely requires complex changes in transcription, protein 
synthesis and metabolism which in principle can all be captured comprehensively by integrated OMICs approaches. However, such in vivo OMICs are hampered by the limited availability of either bacterial mRNA or proteins from such settings. Thus, despite their great potential, only a few transcriptome or proteome studies have so far been performed to investigate the adaptation of $S$. aureus to the host environment following internalization.

Analyzing the transcriptome of S. aureus 6850 in the initial hours after internalization by A549 cells Garzoni et al. observed profound transcriptional adaptation patterns that involved approximately $40 \%$ of all putative ORFs of S. aureus 6850 (Garzoni et al., 2007). In another approach the transcriptome profiles of methicillin-resistant $S$. aureus USA300 were investigated in human abscesses and infected mouse kidneys (Date et al., 2014). That study established that transcriptional adaptations in both models were remarkably similar and included up-regulation of genes coding for multiple proteases and toxins as well as iron and peptide transporters. Furthermore, the specific influence of the global regulators $a g r B$ and saeRS on regulation of virulence associated genes in vivo was examined.

Proteome studies are even more challenging because the large excess of interfering human host proteins complicates identification especially of low abundant bacterial proteins. In a first attempt to investigate the adaptation of $S$. aureus to host cells at the proteome level Miller et al. analyzed the impact of cocultivation with THP-1 macrophages but confined the study to non-adherent, non-internalized $S$. aureus cells (Miller et al., 2011). The comparative study revealed that $S$. aureus NCTC8325 induced the stringent response, activated the alternative sigma factor SigB, enhanced its capability to respond to oxidative stress and produced increased levels of virulence factors including phenol-soluble modulins (PSMs) in response to the presence of macrophages (Miller et al., 2011). Proteome analysis of internalized bacteria requires enrichment by differential centrifugation, immunomagnetic separation, or fluorescent assisted cell sorting. A first analysis of the complex proteome adaptation of S. aureus to the intracellular milieu of human epithelial cells was performed in 2010 (Schmidt et al., 2010). Human bronchial epithelial S9 cells were infected with $S$. aureus HG001 pMV158GFP, which expresses green fluorescent protein constitutively, allowing separation of internalized $S$. aureus from the whole cell lysate by cell sorting prior to LC-MS/MS analysis of the bacterial proteins (Schmidt et al., 2010). In that study, 591 bacterial proteins were identified and 367 could be quantified using stable isotope labeling of amino acids in cell culture (Ong et al., 2002). Using an optimized variant of the original protocol we were now able to identify 1302 bacterial proteins and monitor more than 980 S. aureus proteins quantitatively from as little as 1-2 million internalized bacteria (Pförtner et al., 2014). Such a comparative profiling of the $S$. aureus wild type HG001 and its isogenic $\triangle$ sigB mutant revealed that the alternative sigma factor SigB of $S$. aureus is activated immediately following internalization into S9 cells and that loss of SigB triggered proteome changes reflecting the different residual growth rates of wild type and $\operatorname{Sig} B$ mutant, respectively, the resistance to methicillin, adaptation to oxidative stress and protein quality control mechanisms (Pförtner et al., 2014).
However, the particular response of bacterial pathogens will also depend on the host responses mounted and thus on the host cell line used. Recently, Eisenreich et al. reviewed the variability of host responses either of different cell lines infected with the same pathogen or the same cell line infected with different pathogens (Eisenreich et al., 2013). The data strongly support the notion that the choice of the host cell line or organism crucially influences the impact of bacterial infections. To our knowledge, the variation of adaptive responses of $S$. aureus upon internalization by different host cells has not been addressed yet.

Here, we comparatively analyzed intracellular survival and physiological adaptation of $S$. aureus HG001 to two commonly used human lung epithelial cell lines, A549 (Liang et al., 2009; Wang et al., 2013), and S9 cells (Below et al., 2009; Hermann et al., 2014). Additionally, we used human embryonic kidney HEK 293 cells. This cell line was chosen since it is also an established cell culture model for infections with S. aureus (Sinha et al., 1999; Cucarella et al., 2002; Maya et al., 2012). We characterized the proteome adaptation of $S$. aureus HG001 to the three different cell lines during the initial phase between $2.5 \mathrm{~h}$ and $6.5 \mathrm{~h}$ postinfection (p.i.). Analyzing each $2 \times 10^{6}$ bacteria, 1443 S. aureus proteins, including metabolic enzymes and virulence factors, were identified and quantified by a combination of highly sensitive LCMS/MS approaches, spectral library and classical FASTA database searches in all three cell lines. Thus, analyzing three biologically independent sample series we were able to cover almost $50 \%$ of the total proteome of $S$. aureus HG001 and describe common and specific adaptation processes of $S$. aureus upon internalization by different cell lines. Furthermore, we generated complementing metabolome data for each host cell line representing the nutrient supply available for the bacteria in each cell line.

\section{MATERIALS AND METHODS BACTERIAL STRAIN AND CULTIVATION CONDITIONS}

S. aureus strain HG001 (Herbert et al., 2010) carrying plasmid pMV158GFP (Nieto and Espinosa, 2003) was used throughout the study. Bacteria were cultivated in prokaryotic minimal essential medium (pMEM) until the exponential growth phase $\left(\mathrm{OD}_{600}\right.$ 0.4) as described previously (Depke et al., 2014). The number of bacterial cells present in the culture at $\mathrm{OD}_{600}$ was determined by flow cytometry with a Guava easyCyte ${ }^{\mathrm{TM}}$ flow cytometer (Millipore, Billerica, MA, USA) using GFP constitutively expressed from pMV158GFP.

\section{CELL LINES AND CULTIVATION CONDITIONS}

S9 cells [adeno 12 SV40 hybrid virus-transformed human bronchial epithelial cells, ATCC ${ }^{\circledR}$ number CRL-2778 (Zeitlin et al., 1991; Flotte et al., 1993)] and A549 cells [human lung cancer-derived alveolar epithelial cells, ATCC ${ }^{\circledR}$ number CCL185, (Lieber et al., 1976)] were cultivated in eukaryotic minimal essential medium (eMEM) as described previously (Pförtner et al., 2013). HEK 293 cells [adenovirus type 5-transformed human embryonic kidney cells (Graham et al., 1977)] were cultivated in modified eMEM for HEK 293 cells (HEK-eMEM; $1 \times$ MEM [Biochrom AG), supplemented with additional $10 \%$ $(\mathrm{v} / \mathrm{v})$ dialyzed fetal bovine serum (FBS, Invitrogen), 2\% (v/v) L-glutamine (PAA), $1 \%(\mathrm{v} / \mathrm{v})$ sodium pyruvate (PAA), and $1 \%$ 
(v/v) non-essential amino acids (PAA)]. Cells were cultured in 10 -cm-diameter tissue culture plates at $37^{\circ} \mathrm{C}, 5 \% \mathrm{CO}_{2}$ in a humid atmosphere. Prior to infection experiments, host cells were seeded in 24-well tissue culture plates 3 days in advance and cultivated until confluence.

\section{INTERNALIZATION SETTINGS}

S. aureus HG001 pMV158GFP was cultivated in pMEM until $\mathrm{OD}_{600}$ 0.4. Based on a calibration curve, an infection mix of bacterial culture was prepared in host cell medium buffered with $2.2 \mathrm{~g} / \mathrm{L}$ sodium hydrogen carbonate. Bacterial cultures were diluted in this mix to infect host cells with a multiplicity of infection (MOI) of 25. Afterwards, the cell culture medium of confluent host cells was replaced by this infection mix, and bacteria could sediment, attach to and be internalized by host cells in an incubator at $37^{\circ} \mathrm{C}$ and $5 \% \mathrm{CO}_{2}$ for $1 \mathrm{~h}$. Subsequently, one $\mathrm{mL}$ of host cell supernatant containing non-internalized bacteria was collected to represent the non-adherent control. The remaining infection mix was replaced by host cell medium containing $10 \mu \mathrm{g} / \mathrm{mL}$ lysostaphin (AMBI PRODUCTS LLC, Lawrence, NY, USA). Non-internalized $S$. aureus cells were killed efficiently in this step within $30 \mathrm{~min}$ (Pförtner et al., 2013). After $2.5 \mathrm{~h}$ and $6.5 \mathrm{~h}$ supernatant was removed and cell layers were washed twice with PBS. Host cells were lysed with $0.1 \%$ Triton X-100 in water for $5 \mathrm{~min}$ at $37^{\circ} \mathrm{C}$ and rinsed afterwards with PBS. Bacterial cell counts were again determined with a Guava easyCyte ${ }^{\mathrm{TM}}$ flow cytometer (Millipore).

\section{CELL SORTING AND ON-MEMBRANE DIGESTION}

GFP expressing bacteria from exponentially growing cultures $\left(\mathrm{OD}_{600} 0.4\right)$, non-adherent controls, and internalized bacteria $2.5 \mathrm{~h}$ and $6.5 \mathrm{~h}$ p.i. were sorted from culture or host cell debris using a FACSAria II onto a low protein binding filter membrane $(0.22 \mu \mathrm{m}$ pore size) of a 96 -well microtiter plate (Millipore, Schwalbach, Germany) by applying vacuum (450-550 mbar) to the filter plate to allow constant removal of the fluid (Pförtner et al., 2013). The $488 \mathrm{~nm}$ laser was applied to excite GFP and emitted fluorescence was detected at 515-545 nm (FITC-channel). Two million bacteria were collected per sample. Filters were rinsed with $200 \mu \mathrm{L}$ PBS, and membranes were cut in four pieces and stored at $-20^{\circ} \mathrm{C}$. On-membrane digestion was performed as described earlier using lysostaphin and the protease trypsin and subsequent purification of peptides using $\mathrm{C}_{18}$ ZipTip columns (Merck Millipore, Billerica, MA, USA) (Pförtner et al., 2014).

\section{ANALYSIS OF BACTERIAL PROTEINS BY MASS SPECTROMETRY AND SUBSEOUENT DATA ANALYSIS}

MS analysis was performed on a Q Exactive mass spectrometer (Thermo Fisher Scientific, Waltham, MA, USA) coupled to a TriVersa NanoMate (Advion, Ltd., Harlow, UK) after separation of peptides with a Dionex UltiMate 3000 nano-LC system (Dionex/Thermo Fisher Scientific, Idstein, Germany). Peptides were separated on a $25 \mathrm{~cm}$ analytical column packed with $2 \mu \mathrm{m}$ C18 particles (Acclaim PepMap RSLC, Thermo Scientific) with the help of a linear gradient ranging from 2 to $25 \%$ buffer $(0.1 \%$ $(\mathrm{v} / \mathrm{v})$ acetic acid in acetonitrile). MS data were acquired with a MS scan resolution of 70,000, and the 10 most abundant isotope patterns with charge state $\geq 2$ from the survey scan were subjected to MS/MS analysis with a resolution of 17,5000. Fragmentation was achieved using higher energy collisional dissociation (HCD). Further details are available as Supplementary Material.

Resulting raw data files were converted to mzML format using msconvert. Then, the mzML files were searched using COMET (Eng et al., 2013) and SpectraST (Lam et al., 2007) and processed using Trans-Proteomic Pipeline (Keller and Shteynberg, 2011). The database contained 84,911 human protein entries [complete proteome and VARSPLIC (Kersey et al., 2000)] and 2891 sequences for $S$. aureus. Common contaminants (115 cRAP) and a sequence-shuffled decoy counterpart were added to the database. For COMET, the parent mass error was set to $\pm 50 \mathrm{ppm}$. $\mathrm{N}$-terminal protein acetylation and methionine oxidation were set as variable modifications. The maximum number of missed cleavage sites was set to 2, and number of enzyme termini was set to 1 . For SpectraST, the parent mass error was set to \pm 1.0 Daltons. The spectral library was constructed from previous Q Exactive runs which were searched using COMET with the same parameters using a $S$. aureus protein database only [Michalik et al., unpublished data]. Peptides identified with iProphet probability $\geq 0.9$ were used to construct the spectral library (Shteynberg et al., 2011). The PeptideProphet outputs from both search engines were combined using iProphet.

The reSpect algorithm was applied to identify and attenuate the peaks in the MS/MS spectra that were excluded by the first pass search. A second search round was performed on the reSpect processed spectra, with a mass tolerance matching the selection window of the mass spectrometer and using possible charge states of 1 through 5, which allowed the identification of novel distinct peptide sequences not seen in the single pass analysis. The reSpect searches were analyzed separately from the first pass searches, and also using PeptideProphet and iProphet to establish accurate error rates.

Only peptides with a probability greater than $0.8(\sim$ TPP error rate $<0.01)$ and without missed cleavages were considered for further relative quantitation on protein level. The quantitation was performed using the "MSstats R package for statistical relative quantitation of proteins and peptides" implemented in Skyline package v2.5 (Schilling et al., 2012; Choi et al., 2013). The areas under the curve (AUC) of peptides were summed to obtain single protein intensities. Mean values from three biological replicates were used which were median normalized to the values of non-adherent bacteria control.

The mass spectrometry proteomics data have been deposited to the ProteomeXchange Consortium (Vizcaino et al., 2014) via the PRIDE partner repository with the dataset identifier PXD001003.

Principal component analysis was performed using the Genedata Analyst v7.6 software (Genedata AG, Basel, Switzerland). Median normalized $\log _{10}$ intensity values for all proteins quantified in all cell lines were used for calculation of the variances of the cell lines and sampling points. For each time point data from three independent biological samples were used to calculate average values.

Box blots of functional groups were generated using the SEED database of $S$. aureus NCTC8325 [SEED DB version 2.0 
(Overbeek et al., 2005)], and Voronoi treemaps were created using the Paver software (DECODON GmbH) (Bernhardt et al., 2013).

\section{ANALYSIS OF HOST CELL METABOLOME Sampling and extraction of host cell metabolites}

Confluent cells in $15-\mathrm{cm}$-diameter tissue culture plates were treated for $1 \mathrm{~h}$ with a sterile "infection mix" as described above, imitating a MOI 25 but leaving out bacteria. For this control the $\mathrm{pH}$ was set to 6.9 , corresponding to the $\mathrm{pH}$ of a bacterial culture at $\mathrm{OD}_{600}$ 0.4. Samples of cellular extracts were generated and extracted as described previously (Gierok et al., 2014). In brief, the supernatant was collected separately. After washing with physiological sodium chloride solution ice cold methanol was added to the cells which were subsequently scraped from the plate and transferred into a centrifugation tube. The plate was washed with double distilled water, which was combined with the methanol fraction and frozen in liquid nitrogen. After adding of the internal standards, metabolites were extracted with a methanol:water:chloroform ratio of 5:5:1 and samples were prepared for analytical measurements as described (Gierok et al., 2014).

\section{Acquisition of metabolome data}

GC/MS setup and analysis. Lyophilized samples were derivatized using a two-step derivatization method with MeOX (Sigma-Aldrich) and MSTFA (Chromatographie Service GmbH) (Strelkov et al., 2004). For identification and quantification of metabolites a GC/MS method described previously was applied (Gierok et al., 2014). Qualitative and quantitative analyses were performed using ChromaTOF software (LECO Corporation). The computed metabolite concentrations were further related to the respective cell number.

LC/MS setup and analysis. For identification and quantitation of metabolites a LC/MS method with an ion-pairing reagent and a SymmetryShield RP18 column (Waters) was used with a setup as described (Gierok et al., 2014). Metabolite quantification was performed by QuantAnalysis ${ }^{\circledR}$ (Bruker Daltonik) using previously acquired standard curves. The computed metabolite concentrations were further normalized and related to the respective cell number.

${ }^{1}$ H-NMR analysis of the extracellular medium. Two $\mathrm{mL}$ of the medium were filtered through a $0.22 \mu \mathrm{m}$ syringe sterile filter (Sarstedt AG\&Co) and directly frozen. Qualitative and quantitative data analysis was carried out in a Bruker AVANCE-II 600 NMR spectrometer operated by TOPSPIN 3.1 software and by using AMIX $^{\circledR}$ (Bruker Biospin) as described (Dörries and Lalk, 2013). The NMR-data are available via the MetaboLights database (Haug et al., 2013) under the accession number MTBLS102 (www.ebi.ac.uk/metabolights/MTBLS102).

\section{RESULTS AND DISCUSSION \\ EXPERIMENTAL SETUP AND ANALYSIS WORKFLOW}

It is well established that different cell lines exhibit different protein patterns and adaptation responses that are tailored to their specific physiological role within the human body (Schirle et al., 2003). Therefore, it is of interest to comparatively profile bacterial adaptation after internalization by different cell line types such as S9, A549, and HEK 293. S. aureus HG001 was grown until $\mathrm{OD}_{600} 0.4$ and transferred to human cell lines grown to confluence. After $2.5 \mathrm{~h}$ and $6.5 \mathrm{~h}$ the internalized bacteria were isolated via cell sorting (Pförtner et al., 2014) and subjected to analysis by mass spectrometry. Time resolved proteome patterns of internalized S. aureus HG001 were compared with the protein profiles of the non-adherent control bacteria that were exposed to human host cells but were not internalized. This control was chosen because previous transcriptome studies have established that $S$. aureus only mounts adaptation reactions after internalization but not after mere contact with human non-professional phagocytic cells (Garzoni et al., 2007).

In order to support the proteome data we further investigated the metabolome profile of the human host cells and the corresponding culture supernatant to elucidate metabolic shifts and available nutrients (Figure 1A). Whereas the supernatants of all three human cell lines displayed similar metabolite profiles, some metabolites were detected in different amounts in the intracellular metabolome of the three cell lines (Supplementary Material Figure 1).

\section{INTRACELLULAR SURVIVAL AND REPLICATION}

Fluorescence microscopy and flow cytometry revealed an intracellular replication of $S$. aureus in all three cell lines as well as formation of clusters at $6.5 \mathrm{~h}$ p.i. (Supplementary Material Figure 2). In all three cell lines, only a rather small proportion of host cells ranging from $10-20 \%$ was indeed infected at an MOI of 25. Intracellular growth of S. aureus HG001 differed when internalized by different cell types (Figure 1B). The number of intracellular bacteria started to increase immediately after internalization by A549 and S9 cells and was finally approximately threefold higher $6.5 \mathrm{~h}$ p.i.. In contrast, numbers of internalized bacteria remained almost constant for up to $3.5 \mathrm{~h}$ p.i. in HEK 293 and then increased more rapidly in the subsequent $3 \mathrm{~h}$ to finally reach a level almost eightfold higher than $1.5 \mathrm{~h}$ p.i. (Figure 1B). Considering these values, we assumed that the different growth behavior might be reflected in the proteome adaptation of $S$. aureus HG001 upon internalization.

\section{PROTEOME ANALYSIS OF INTERNALIZED $S$. AUREUS Staphylococcal protein identification and quantitation}

The main limitation of host-pathogen proteomics approaches is the low number of available bacteria and the large excess of human host proteins. Due to this fact, it is still a challenge to reliably identify $S$. aureus specific peptides in such a complex mixture. That is why we first applied a COMET search using a decoy database of $S$. aureus HG001 combined with human Uniprot/Swissprot DB and identified 1393 staphylococcal proteins in total with a peptide probability greater than $0.8(\sim \mathrm{FDR}<$ 0.01 ). Finally, a spectral library comparison using a $S$. aureus HG001 specific database [Depke et al., unpublished data] complemented with a reSpect search was applied (Figure 2). Taken all identifications together, 1484 proteins were finally identified with an FDR less than 0.01 .

With respect to quantitation, 1443 staphylococcal proteins could also be quantified over time for all three cell line models (Figure 2, Supplementary Material Table 1). With the improved 

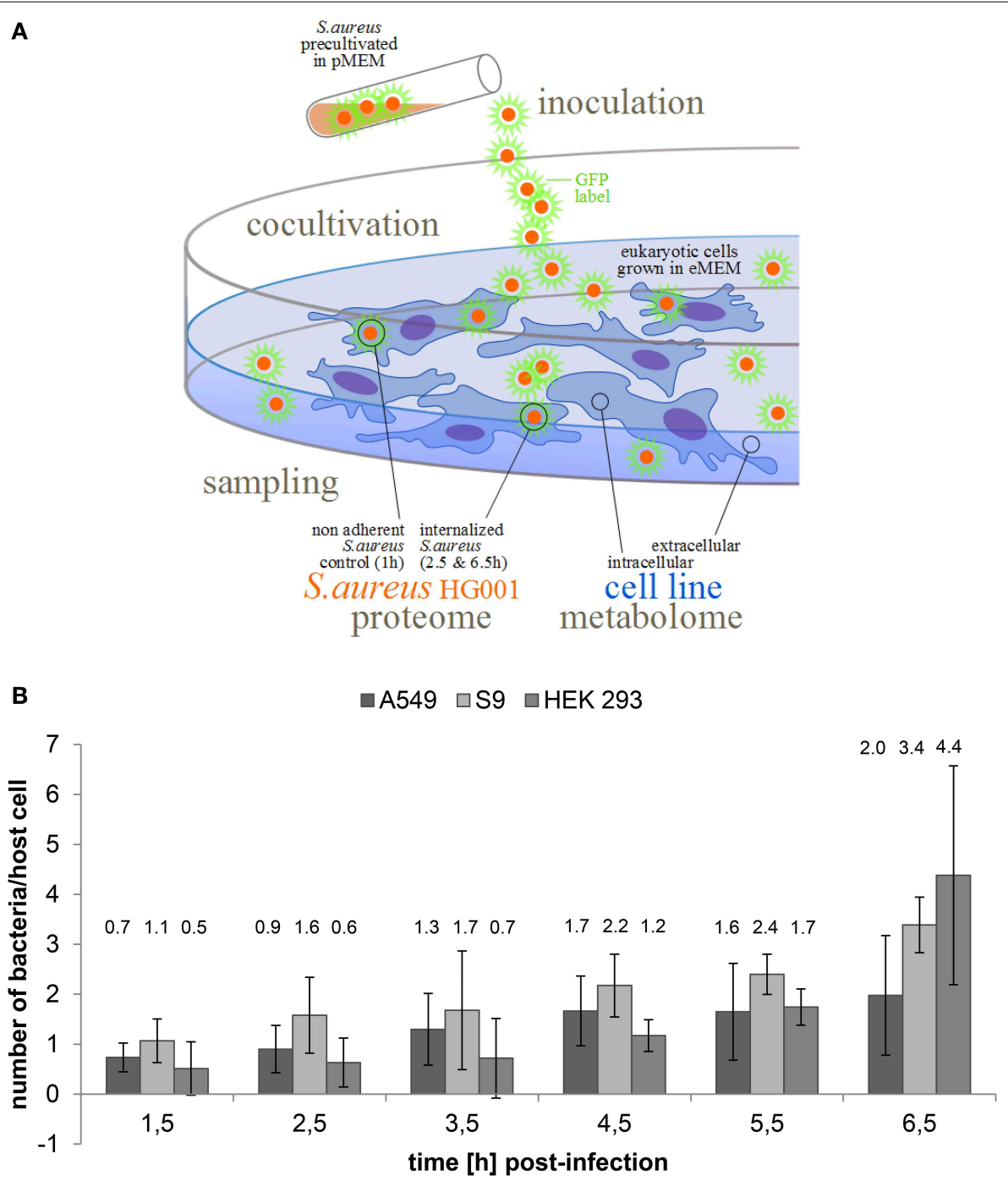

FIGURE 1 | Internalization of $S$. aureus HG001 by human cell lines. (A) Experimental course of action. GFP expressing bacteria were cultivated in pMEM until $\mathrm{OD}_{600} 0.4$ (exponential growth). Human cell lines (S9, A549, and HEK 293) were cultivated in eMEM and infected with $S$. aureus HG001 pMV158GFP cells. The proteome of the non-adherent and the internalized bacteria was investigated after cell sorting via flow cytometry.
In parallel, the metabolome of non-infected human cells and the cell culture supernatant was recorded. (B) Number of internalized bacteria per host cell. GFP expressing bacteria were counted by flow cytometry from lysed host cells. For all three cell lines time-resolved bacterial counts are provided. Results represent the mean and standard deviation of three biological replicates. settings in both mass spectrometry and data analysis we were now able to quantify about $50 \%$ of the whole proteome (2891 proteins in S. aureus NCTC8325 database) from 2 million S. aureus HG001 cells after internalization and therefore provide a comprehensive description of the behavior of this important pathogen during infection. This is roughly three times more compared to a first proteome study of internalized S. aureus HG001 (Schmidt et al., 2010).

In order to gain a first inside into the global response of S. aureus to internalization by three different host cell lines a principal component analysis (PCA) plot of three sampling points (non-adherent control, $2.5 \mathrm{~h}$ and $6.5 \mathrm{~h}$ p.i.) was generated (Figure 3). For this analysis, the median-normalized data of all quantified proteins were taken into account. All samples from internalized bacteria, both at $2.5 \mathrm{~h}$ and $6.5 \mathrm{~h}$ p.i., were clearly separated from the non-adherent controls by the first component. Remarkably, the protein pattern of $S$. aureus internalized by HEK 293 kidney cells for $6.5 \mathrm{~h}$ was clearly separated in the second component from the other two $S$. aureus samples internalized by epithelial cell lines for the same time period, which indicates a different behavior of S. aureus inside the HEK 293 cells. The Voronoi treemaps displayed in Figure 4 provide a protein-resolved picture of the proteome adaptation where proteins which are assigned to the same biochemical pathway or adaptation reaction are grouped together (legends in Figures 4A,E). Again the patterns of $S$. aureus internalized by epithelial cells (S9 or A459) look more similar to each other than to HEK293-internalized S. aureus. Exemplarily one can focus on protein biosynthesis which appears more reduced in level in comparison to the control in $S$. aureus originating from A549 (Figure 4B) and S9 (Figure 4C) compared 


\section{internalization of $S$. aureus $\mathrm{HG00}$ \\ by A549, S9, or HEK 293 cells \\ cell sorting \\ tryptic on-filter digestion \\ nLC-MS/MS Q Exactive \\ COMET search vs combined \\ S. aureus and human Sprot \\ FASTA-DB (decoyed) \\ SpectraST search $v$ s}

S. aureus specific spectral library

reSpect search of remaining spectra vs $S$. aureus

calculation of final iProphet from COMET, SpectraST, and reSpect

calculation of AUC of each run by

Skyline, quantitation by

Genedata Analyst

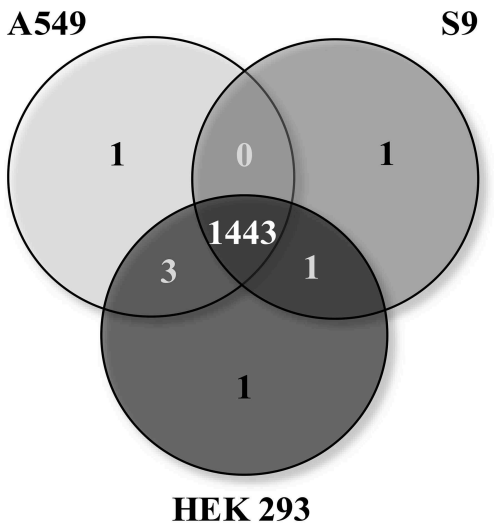

FIGURE 2 | Proteomics identification and quantitation workflow. Bacteria were sorted from host cell debris onto membrane filters by flow cytometry. Subsequently, bacterial proteins were digested on-filter using trypsin prior to $\mathrm{nLC}-\mathrm{MS} / \mathrm{MS}$ acquisition with a $\mathrm{Q}$ Exactive. Database analysis was performed in three steps: (i) COMET search against human and S. aureus HG001 Sprot database, (ii) SpectraST against a specific S. aureus HG001 spectral library, and (iii) reSpect search of non-matching spectra. Calculation of AUC of peptides and proteins exceeding an iProphet score of 0.8 was performed with Skyline and the Genedata Analyst. The Venn diagram represents all proteins which were detected in any of the three biological replicates for each host cell line.

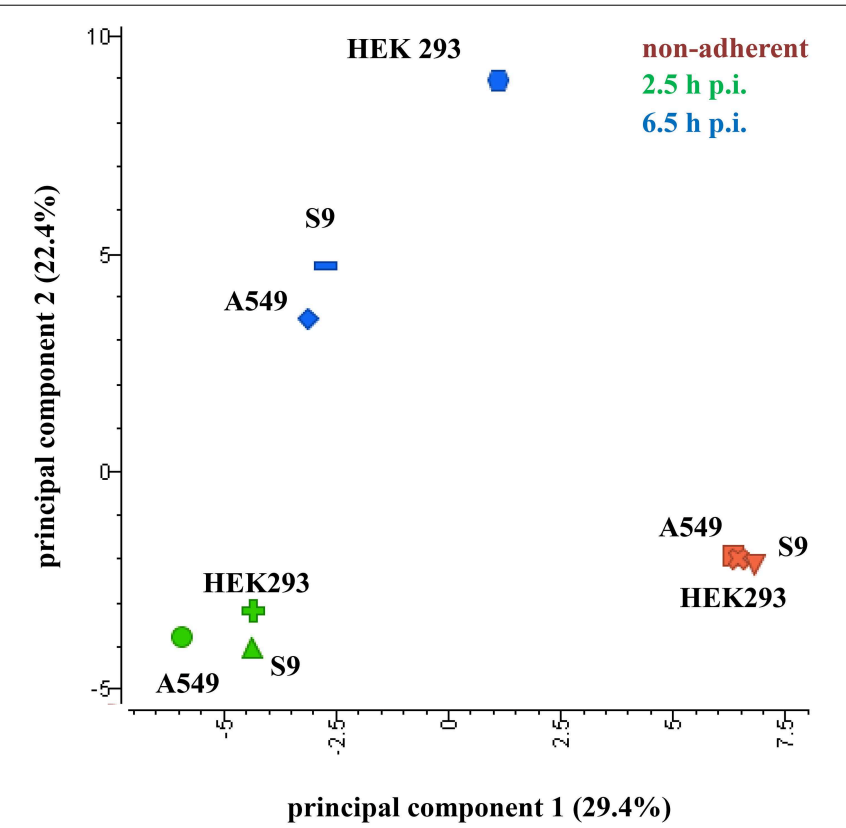

FIGURE 3 | Principal component analysis of $\boldsymbol{S}$. aureus proteins. Samples were separated by the cell lines (component 1, 29.4\% variance) as well as the different treatment groups (component 2, $22.4 \%$ variance). Only proteins with quantitative data for all samples, (median normalized AUC data, probability $>0.8$ ) were considered for the analysis. Data from three biological replicates are represented.

to those isolated 2.5 p.i. from HEK 293 cells (Figure 4D). On the contrary, proteins involved in central carbon metabolism tend to be in general more strongly induced in S. aureus from A549 (Figure 4F) and S9 (Figure 4G) cells than in S. aureus from HEK 293 cells 6.5 h p.i. (Figure $4 \mathbf{H}$ ).

\section{Common reaction of $S$. aureus proteome to internalization by three different host cells}

In order to characterize $S$. aureus specific pathways or reactions which were similar after internalization by the three different host cell lines, we plotted the $\log _{2}$ intensities of the proteins belonging to these pathways in a box-plot. For this analysis, the third level of the functional category level from SEED was used (Supplementary Material Table 2) which combines several proteins which participate in the same functional pathways and is thus more robust than analysis at the level of individual proteins.

Presumably, S. aureus displays significantly reduced growth rates after internalization by host cells compared to those possible in the cell culture medium alone. In agreement with this assumption we observed decreased levels of proteins composing the large (ribosome LSU bacterial, 29 of 35, Figure 5A) and small (ribosome SSU bacterial, 20 of 21, Figure 5B) subunit of the ribosome during the first $6.5 \mathrm{~h}$ of internalization into all three cell lines. Furthermore, proteins involved in de novo purine biosynthesis ( 15 of 15 , Figure $5 \mathrm{C}$ ) and ribonucleotide reduction (4 of 7 , Figure 5D) were also decreased in level probably reflecting the lower levels required to sustain lower growth rates. The level of cold shock proteins (Figure 5E) was also commonly reduced after 


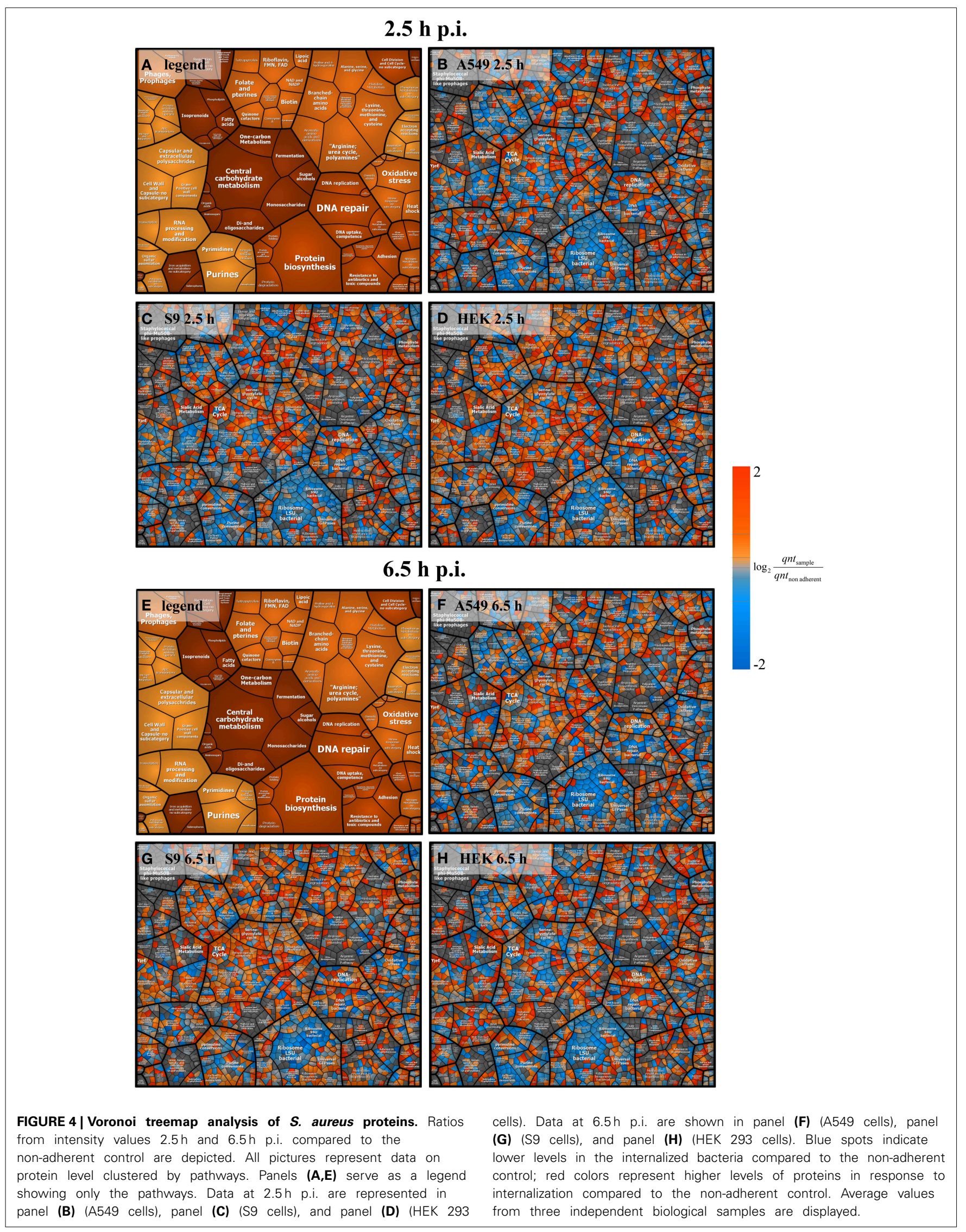




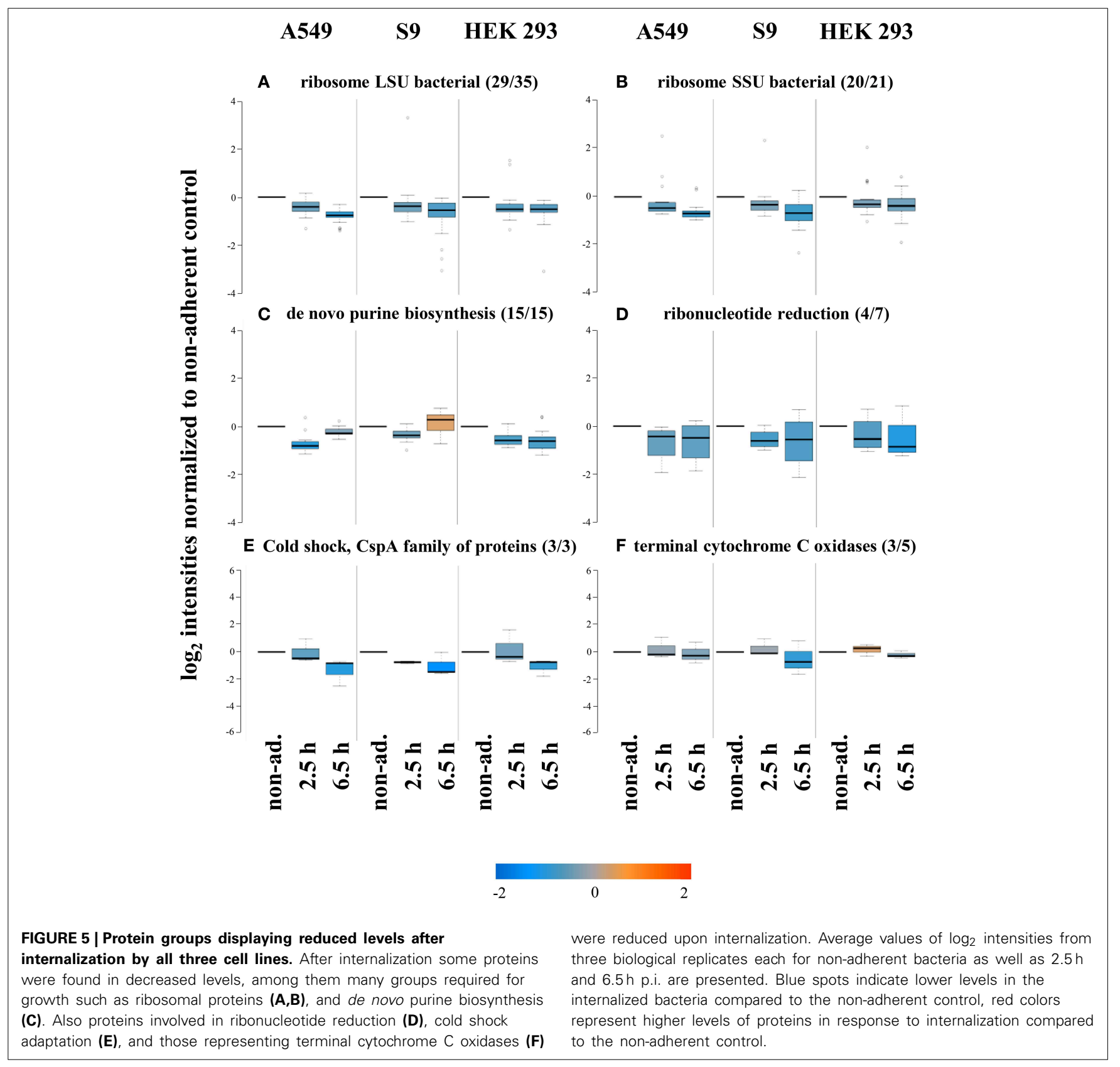

internalization compared to that in non-adherent control cells, but the physiological rationale for this observation is currently not clear.

Interestingly, the level of components of the cytochrome C oxidase ( 3 of 5) was also reduced in internalized bacteria vs. the non-adherent control (Figure 5F). This regulation possibly indicates an adaptation to reduced oxygen levels inside host cells, because this main terminal oxidase is preferentially used under aerobic conditions (Götz and Mayer, 2013) and likely replaced by a specialized oxidase under the likely microaerobic conditions inside host cells.

Because S. aureus also needs to adapt its protein inventory to the special conditions of the intracellular environment, we looked for pathways commonly displaying increased protein levels after entering the different cell lines, compared to non-adherent control bacteria exposed to the same medium and thus nutrient supply conditions. Some amino acid biosynthesis pathways, such as arginine (5 of 14, Figure 6A) and lysine ( 9 of 11, Figure 6B) biosynthesis, displayed increased protein levels for all cell lines which might be an adaptation to lower amino acids levels inside host cells vs. the cell culture medium. Having the metabolome data at hand, we wanted to make an effort to validate this hypothesis. For A549 cells we could directly compare the intracellular and extracellular concentrations because the cell volume of these cells has previously been reported (Jiang et al., 2010). However, intracellular lysine levels of uninfected host cells were similar to those in the supernatant, and arginine levels could not be measured because of technical reasons. Metabolite levels might differ 


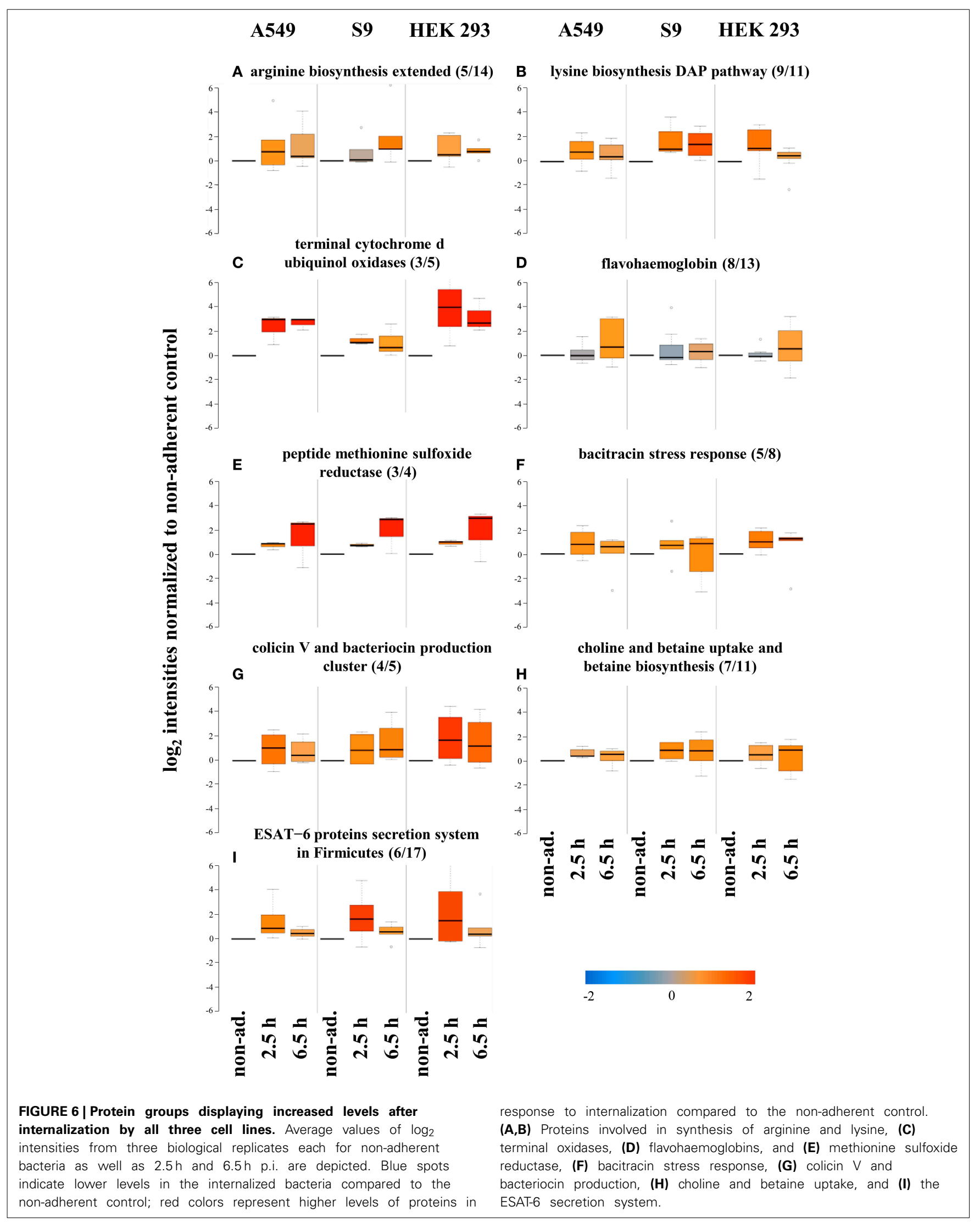


in infected host cells, but since only a small proportion of host cells indeed carried $S$. aureus we could not assess metabolite levels in this sub-group specifically. Striking differences between the extracellular and host cell concentrations were observed for glycine (intracellular $2.3 \mathrm{mmol} / \mathrm{L}$, extracellular $0.1 \mathrm{mmol} / \mathrm{L}$ ), threonine (intracellular $4.7 \mathrm{mmol} / \mathrm{L}$, extracellular $0.9 \mathrm{mmol} / \mathrm{L}$ ), and glutamate (intracellular $16.0 \mathrm{mmol} / \mathrm{L}$, extracellular $0.5 \mathrm{mmol} / \mathrm{L}$ ) (see Supplementary Material Figure 1).

Levels of subunits of the terminal cytochrome $d$ ubiquinol oxidases (3 of 5) were also higher in internalized $S$. aureus vs. the non-adherent controls (Figure 6C). This observation fits very well to the adaptation of the repertoire of terminal oxidases to the level of oxygen available. While levels of the main oxidase
Qox decreased because this enzyme is preferentially used during oxygen excess (see above), the level of the alternative oxidase $\mathrm{CydAB}$ increased because this enzyme complex is used as an alternative during microaerobic conditions (Götz and Mayer, 2013), thus supporting the notion of a microaerobic environment inside host cells. Furthermore, increases in the levels of flavohaemoglobin ( 8 of 13, Figure 6D), peptide methionine sulfoxide reductase ( 3 of 4 , Figure $6 \mathrm{E}$ ), and bacitracin stress response ( 5 of 8 , Figure $6 \mathrm{~F}$ ) were observed. The same was true for proteins involved in the colicin $\mathrm{V}$ and bacteriocin production clusters ( 4 of 5, Figure 6F) and choline and betaine uptake and betaine biosynthesis (7 of 11, Figure 6G). Also proteins belonging to the ESAT-6 protein secretion system in Firmicutes (6 of 17, Figure 6I) were

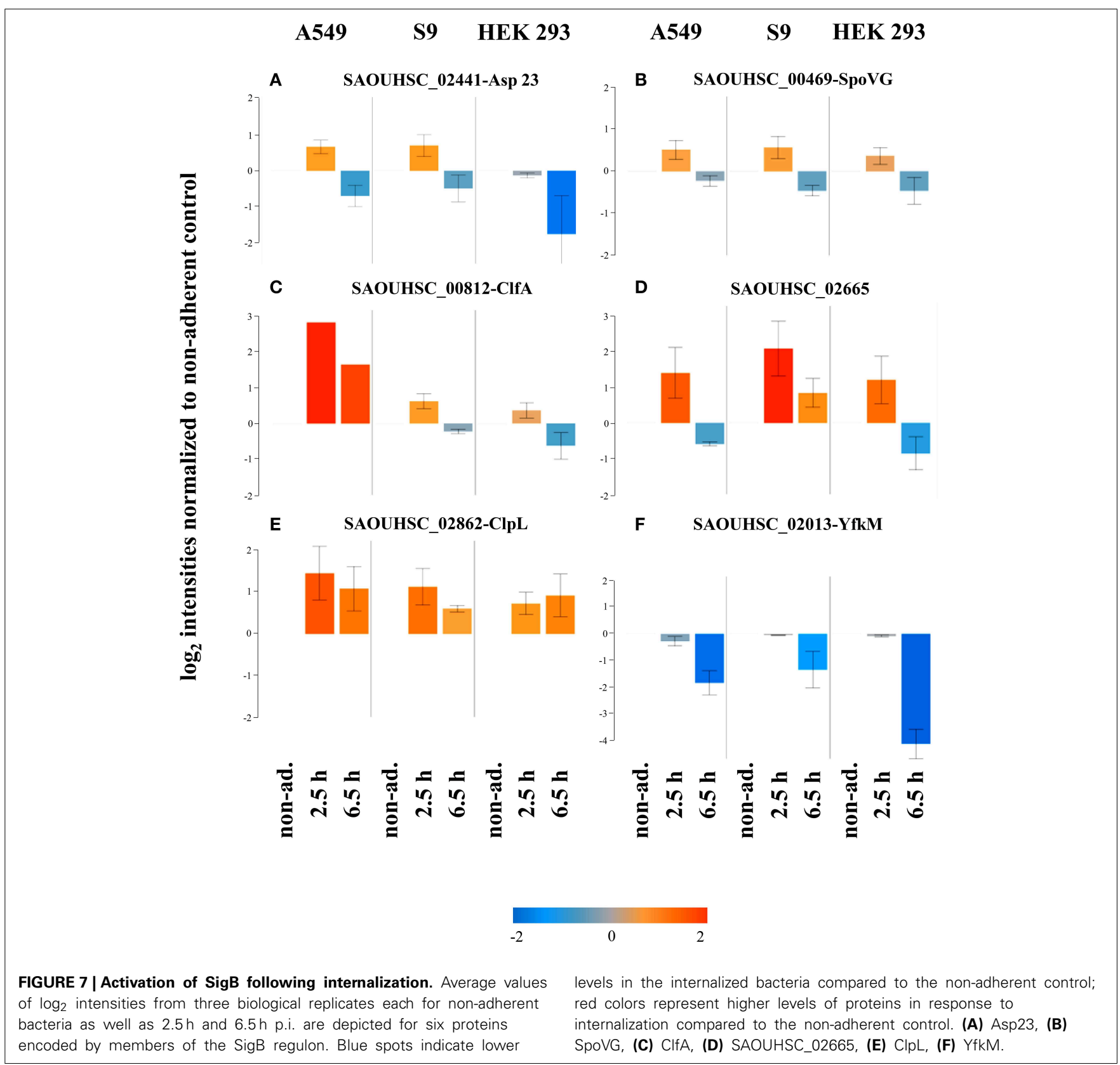


increased in level upon internalization. These results likely reflect adaptations to the hostile host environment. These observations fit very well to those made for individual cell lines (Schmidt et al., 2010) indicating that these responses are more universal.

\section{Activation of the alternative sigma factor SigB upon internalization}

It has recently been shown that the alternative sigma factor SigB which controls many $S$. aureus genes also with impact on virulence is activated following internalization of $S$. aureus HG001 by S9 cells (Pförtner et al., 2014). Since reports on the role of the alternative sigma factor SigB in different animal models and cell culture settings differ (Jonsson et al., 2004; Depke et al., 2012), we wanted to assess if the activation described for S9 epithelial cells is also conserved upon internalization by A549 and HEK 293 cells. The data presented in Figure 7 support this notion. Of the six proteins for which Pförtner et al. (2014) have clearly shown SigB-dependent increases in level following internalization of $S$. aureus HG001 by S9 cells, five [Asp23 (Figure 7A), SpoVG (Figure 7B), CflA (Figure 7C), ClpL (Figure 7D), and SAOUHSC_02665 (Figure 7F)] displayed increases in level following internalization that were maintained for ClpL even $6.5 \mathrm{~h}$ after internalization. The increase in protein content was observed following internalization by all three cell lines, even if the particular patterns sometimes differed. The increase in level could not be confirmed for YfkM (Figure 7F).

\section{Differences in the adaptation of S. aureus to internalization by different types of host cell lines}

In this study, the focus lies on the identification of conserved and specific responses of $S$. aureus HG001 to the three different cell lines, with the HEK 293 cell line constituting an additional host model for the internalization setup in comparison with the two lung epithelial cell lines (S9 and A549). As indicated by the different growth pattern (Figure 1B) and the PCA plot (Figure 3) the strongest differences were observed in the HEK 293 cells.

Here we provide information for selected protein classes and pathways which were differentially regulated depending on the host cell model. Although S. aureus HG001 showed similar adaptation patterns over time for all three internalization models, some distinct differences were observed.

Different patterns were for example observed for enzymes involved in threonine degradation in HEK 293 cells compared to the two epithelial cell lines (Figure 8A). These differences coincided with differences in the host cell levels of threonine (Supplementary Material Figure 1). The intracellular levels of threonine and the degrading enzymes (5 of 5) were higher in HEK 293 cells. A second example concerns enzymes involved in fermentation (9 of 9), which were present in lower levels in S9 cells compared to $S$. aureus internalized by either A549 or HEK 293 cells (Figure 8B). More specifically, enzymes such as alcohol dehydrogenase (Adh1), L-lactate dehydrogenase (LctE), L-lactate dehydrogenase 2 (Ldh2), and D-lactate dehydrogenase (Ddh) increased in level after internalization by A549 and HEK293 within 6.5 h up to 2-3fold (Ldh2 and Ddh) or 8-10fold (Adh1 and LctE), respectively (Supplementary Material Figure 3A), indicating the supplementary utilization of fermentative enzymes under microaerobic conditions.

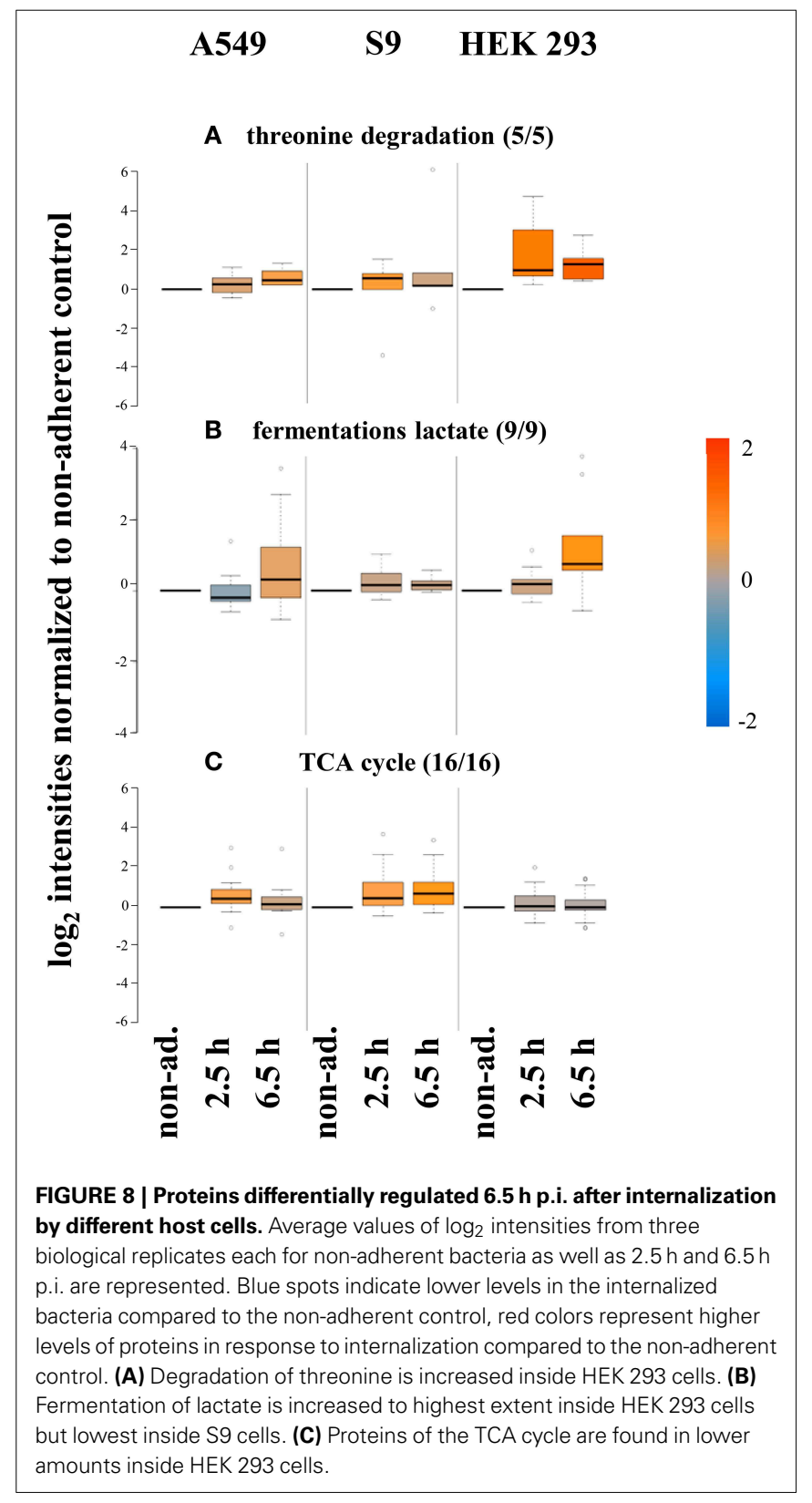

The third example is the tricarboxylic acid cycle (TCA). Most of the enzymes of the TCA cycle (16 of 16) that could be monitored in our proteomics approach increased in level in $S$. aureus following internalization by all three cell lines (Figure 8C). This increase probably reflects a switch from glucose to alternative energy-/carbon sources, which depends on increased TCA cycle capacity in internalized bacteria compared to the non-internalized controls. Astonishingly, a number of TCA cycle enzymes including citrate synthase (CitZ), isocitrate dehydrogenase (CitC), succinyl-CoA synthetase subunit beta (SucC) and succinate dehydrogenase flavoprotein subunit (SdhA) increased in level upon internalization by epithelial cells but not in S. aureus internalized by HEK293 cells (Supplementary Material Figure 3B). 
These selected examples suggest that $S$. aureus can on the one side adapt its physiology to the intracellular life style by conserved adaptation reactions but on the other side also has the capacity to respond to subtle differences encountered in the different host cell niches. Quantitative information about further proteins analyzed in this approach as well as their classification to functional groups is provided as Supplementary Material Tables1, 2.

\section{CONCLUSION}

With this study we provide the first time quantitative data for about $50 \%$ of the predicted $S$. aureus HG001 proteins from as little as two million internalized $S$. aureus bacteria. The remarkable increase of quantifiable proteins is the result of mass spectrometry applying highly sensitive instruments and utilization of new algorithms for peptide identification. This optimized pipeline allowed us to comprehensively elucidate the early phase of the adaptational response of $S$. aureus after entering human epithelial or kidney cells.

Observations made for specific cell line settings such as the decrease in content of ribosomal proteins or proteins belonging to the de novo purine biosynthesis or the increase of proteins belonging to stress responses were now shown to be conserved adaptations reactions to the intracellular environment of human host cells in general.

In addition to common mechanisms of adaptation, we also report differences between $S$. aureus cells internalized by the epithelial cell lines A549 or S9 and the kidney cell line HEK 293, especially $6.5 \mathrm{~h}$ p.i., e.g., in threonine degradation as well as level of fermentation enzymes and TCA cycle. Complementing the proteome analysis with metabolomics measurements, we also linked some of the observed adaptation reactions to the altered nutrient supply encountered inside of human host cells. It will now be interesting to extend this studies to extended time courses in which $S$. aureus physiology shifts to the well-known "small colony variants," a strategy to survive intracellularly (Kahl, 2014) or to study adaptation to survival inside professional phagocytic host cells.

\section{AUTHOR CONTRIBUTIONS}

Kristin Surmann: Design and performance of wet-lab experiments, data analysis, and writing of manuscript. Stephan Michalik: Data analysis and visualization. Petra Hildebrandt: Design and performance of wet-lab experiments. Philipp Gierok: Metabolome analysis, and writing of manuscript. Maren Depke: Data analysis and writing. Lars Brinkmann: Data analysis. Jörg Bernhardt: Data visualization. Manuela G. Salazar: Data acquisition by mass spectrometry. Zhi Sun: Data analysis. David Shteynberg: Data analysis. Ulrike Kusebauch: Data analysis. Robert L. Moritz: Data analysis. Bernd Wollscheid: Data analysis. Michael Lalk: Supervision of metabolome analysis, writing of manuscript. Uwe Völker: Concept and design of experiments, writing of manuscript. Frank Schmidt: Concept and design of experiments, data analysis, and writing of manuscript.

\section{ACKNOWLEDGMENTS}

The authors thank Kirsten Bartels for excellent help in performing the experiments, Leif Steil and Stefan Weiß for assistance in data analysis, and Bhanu Sinha for provision of HEK 293 cells. The work was financially supported by the BMBF/"Unternehmen Region" as part of the ZIK-FunGene (Uwe Völker, Frank Schmidt), as well as within the framework of the SFB Transregio 34 (Uwe Völker). This work has been funded in part with US federal funds from National Science Foundation Major Research Instrumentation Grant 0923536, by funds from the American Recovery and Reinvestment Act through Grant RC2 HG005805 from the National Human Genome Research Institute, and Grant S10 RR027584, R01 GM087221, Center for Systems Biology/2P50 GM076547 from the National Institute of General Medical Sciences."

\section{SUPPLEMENTARY MATERIAL}

The Supplementary Material for this article can be found online at: http://www.frontiersin.org/journal/10.3389/fmicb. 2014.00392/abstract

\section{REFERENCES}

Almeida, R. A., Matthews, K. R., Cifrian, E., Guidry, A. J., and Oliver, S. P. (1996). Staphylococcus aureus invasion of bovine mammary epithelial cells. J. Dairy Sci. 79, 1021-1026. doi: 10.3168/jds.S0022-0302(96)76454-8

Below, S., Konkel, A., Zeeck, C., Müller, C., Kohler, C., Engelmann, S., et al. (2009). Virulence factors of Staphylococcus aureus induce Erk-MAP kinase activation and c-Fos expression in S9 and 16HBE14o- human airway epithelial cells. Am. J. Physiol. Lung Cell. Mol. Physiol. 296, L470-L479. doi: 10.1152/ajplung.90498.2008

Bernhardt, J., Michalik, S., Wollscheid, B., Völker, U., and Schmidt, F. (2013). Proteomics approaches for the analysis of enriched microbial subpopulations and visualization of complex functional information. Curr. Opin. Biotechnol. 24, 112-119. doi: 10.1016/j.copbio.2012.10.009

Choi, M., Chang, C. Y., and Vitek, O. (2013). MSstats: Protein Significance Analysis in LC-MS, SRM and DIA for Label-free or Label-based Proteomics Experiments. $\mathrm{R}$ package version 2.0.1.

Cucarella, C., Tormo, M. A., Knecht, E., Amorena, B., Lasa, I., Foster, T. J., et al. (2002). Expression of the biofilm-associated protein interferes with host protein receptors of Staphylococcus aureus and alters the infective process. Infect. Immun. 70, 3180-3186. doi: 10.1128/IAI.70.6.3180-3186.2002

Date, S. V., Modrusan, Z., Lawrence, M., Morisaki, J. H., Toy, K., Shah, I. M., et al. (2014). Global gene expression of methicillin-resistant Staphylococcus aureus USA300 during human and mouse infection. J. Infect. Dis. 209, 1542-1550. doi: 10.1093/infdis/jit668

Depke, M., Burian, M., Schäfer, T., Bröker, B. M., Ohlsen, K., and Völker, U. (2012). The alternative sigma factor B modulates virulence gene expression in a murine Staphylococcus aureus infection model but does not influence kidney gene expression pattern of the host. Int. J. Med. Microbiol. 302, 33-39. doi: 10.1016/j.ijmm.2011.09.013

Depke, M., Surmann, K., Hildebrandt, P., Jehmlich, N., Michalik, S., Stanca, S. E., et al. (2014). Labeling of the pathogenic bacterium Staphylococcus aureus with gold or ferric oxide-core nanoparticles highlights new capabilities for investigation of host-pathogen interactions. Cytometry A 85, 140-150. doi: 10.1002/cyto.a. 22425

Dörries, K., and Lalk, M. (2013). Metabolic footprint analysis uncovers strain specific overflow metabolism and D-isoleucine production of Staphylococcus aureus COL and HG001. PLoS ONE 8:e81500. doi: 10.1371/journal.pone.0081500

Eisenreich, W., Heesemann, J., Rudel, T., and Goebel, W. (2013). Metabolic host responses to infection by intracellular bacterial pathogens. Front. Cell. Infect. Microbiol. 3:24. doi: 10.3389/fcimb.2013.00024

Eng, J. K., Jahan, T. A., and Hoopmann, M. R. (2013). Comet: an opensource MS/MS sequence database search tool. Proteomics 13, 22-24. doi: 10.1002/pmic.201200439

Flotte, T. R., Afione, S. A., Solow, R., Drumm, M. L., Markakis, D., Guggino, W. B., et al. (1993). Expression of the cystic fibrosis transmembrane conductance regulator from a novel adeno-associated virus promoter. J. Biol. Chem. 268, 3781-3790. 
Garzoni, C., Francois, P., Huyghe, A., Couzinet, S., Tapparel, C., Charbonnier, Y., et al. (2007). A global view of Staphylococcus aureus whole genome expression upon internalization in human epithelial cells. BMC Genomics 8:171. doi: 10.1186/1471-2164-8-171

Garzoni, C., and Kelley, W. L. (2009). Staphylococcus aureus: new evidence for intracellular persistence. Trends Microbiol. 17, 59-65. doi: 10.1016/j.tim.2008.11.005

Gierok, P., Harms, M., Richter, E., Hildebrandt, J. P., Lalk, M., Mostertz, J., et al. (2014). Staphylococcus aureus alpha-toxin mediates general and cell typespecific changes in metabolite concentrations of immortalized human airway epithelial cells. PLoS ONE 9:e94818. doi: 10.1371/journal.pone.0094818

Götz, F., and Mayer, S. (2013). Both terminal oxidases contribute to fitness and virulence during organ-specific Staphylococcus aureus colonization. MBio 4, e00976-e00913. doi: 10.1128/mBio.00976-13

Graham, F. L., Smiley, J., Russell, W. C., and Nairn, R. (1977). Characteristics of a human cell line transformed by DNA from human adenovirus type 5. J. Gen. Virol. 36, 59-74. doi: 10.1099/0022-1317-36-1-59

Haslinger-Löffler, B., Wagner, B., Brück, M., Strangfeld, K., Grundmeier, M., Fischer, U., et al. (2006). Staphylococcus aureus induces caspase-independent cell death in human peritoneal mesothelial cells. Kidney Int. 70, 1089-1098. doi: 10.1038/sj.ki.5001710

Haug, K., Salek, R. M., Conesa, P., Hastings, J., de Matos, P., Rijnbeek, M., et al. (2013). MetaboLights-an open-access general-purpose repository for metabolomics studies and associated meta-data. Nucleic Acids Res. 41, D781-D786. doi: 10.1093/nar/gks1004

Herbert, S., Ziebandt, A. K., Ohlsen, K., Schafer, T., Hecker, M., Albrecht, D., et al. (2010). Repair of global regulators in Staphylococcus aureus 8325 and comparative analysis with other clinical isolates. Infect. Immun. 78, 2877-2889. doi: 10.1128/IAI.00088-10

Hermann, I., Räth, S., Ziesemer, S., Volksdorf, T., Dress, R. J., Gutjahr, M., et al. (2014). Staphylococcus aureus-hemolysin a disrupts cell-matrix adhesions in human airway epithelial cells. Am. J. Respir. Cell Mol. Biol. doi: 10.1165/rcmb.2014-0082OC. [Epub ahead of print].

Hudson, M. C., Ramp, W. K., Nicholson, N. C., Williams, A. S., and Nousiainen, M. T. (1995). Internalization of Staphylococcus aureus by cultured osteoblasts. Microb. Pathog. 19, 409-419. doi: 10.1006/mpat.1995.0075

Jiang, R. D., Shen, H., and Piao, Y. J. (2010). The morphometrical analysis on the ultrastructure of A549 cells. Rom. J. Morphol. Embryol. 51, 663-667.

Jonsson, I. M., Arvidson, S., Foster, S., and Tarkowski, A. (2004). Sigma factor B and RsbU are required for virulence in Staphylococcus aureus-induced arthritis and sepsis. Infect. Immun. 72, 6106-6111. doi: 10.1128/IAI.72.10.6106-6111.2004

Kahl, B. C. (2014). Small colony variants (SCVs) of Staphylococcus aureusa bacterial survival strategy. Infect. Genet. Evol. 21, 515-522. doi: 10.1016/j.meegid.2013.05.016

Keller, A., and Shteynberg, D. (2011). Software pipeline and data analysis for MS/MS proteomics: the trans-proteomic pipeline. Methods Mol. Biol. 694, 169-189. doi: 10.1007/978-1-60761-977-2_12

Kersey, P., Hermjakob, H., and Apweiler, R. (2000). VARSPLIC: alternativelyspliced protein sequences derived from SWISS-PROT and TrEMBL. Bioinformatics 16, 1048-1049. doi: 10.1093/bioinformatics/16.11.1048

Lam, H., Deutsch, E. W., Eddes, J. S., Eng, J. K., King, N., Stein, S. E., et al. (2007). Development and validation of a spectral library searching method for peptide identification from MS/MS. Proteomics 7, 655-667. doi: 10.1002/pmic.200600625

Liang, X., Yan, M., and Ji, Y. (2009). The H35A mutated alpha-toxin interferes with cytotoxicity of staphylococcal alpha-toxin. Infect. Immun. 77, 977-983. doi: 10.1128/IAI.00920-08

Lieber, M., Smith, B., Szakal, A., Nelson-Rees, W., and Todaro, G. (1976). A continuous tumor-cell line from a human lung carcinoma with properties of type II alveolar epithelial cells. Int. J. Cancer 17, 62-70. doi: 10.1002/ijc.2910170110

Lowy, F. D. (1998). Staphylococcus aureus infections. N. Engl. J. Med. 339, 520-532 doi: 10.1056/NEJM199808203390806

Maya, S., Indulekha, S., Sukhithasri, V., Smitha, K. T., Nair, S. V., Jayakumar, R., et al. (2012). Efficacy of tetracycline encapsulated O-carboxymethyl chitosan nanoparticles against intracellular infections of Staphylococcus aureus. Int. J. Biol. Macromol. 51, 392-399. doi: 10.1016/j.ijbiomac.2012.06.009

Miller, M., Dreisbach, A., Otto, A., Becher, D., Bernhardt, J., Hecker, M., et al. (2011). Mapping of interactions between human macrophages and Staphylococcus aureus reveals an involvement of MAP kinase signaling in the host defense. J. Proteome Res. 10, 4018-4032. doi: 10.1021/pr200224x
Nieto, C., and Espinosa, M. (2003). Construction of the mobilizable plasmid pMV158GFP, a derivative of pMV158 that carries the gene encoding the green fluorescent protein. Plasmid 49, 281-285. doi: 10.1016/S0147-619X(03) 00020-9

Ong, S. E., Blagoev, B., Kratchmarova, I., Kristensen, D. B., Steen, H., Pandey, A., et al. (2002). Stable isotope labeling by amino acids in cell culture, SILAC, as a simple and accurate approach to expression proteomics. Mol. Cell. Proteomics 1, 376-386. doi: 10.1074/mcp.M200025-MCP200

Overbeek, R., Begley, T., Butler, R. M., Choudhuri, J. V., Chuang, H. Y., Cohoon, M., et al. (2005). The subsystems approach to genome annotation and its use in the project to annotate 1000 genomes. Nucleic Acids Res. 33, 5691-5702. doi: 10.1093/nar/gki866

Pförtner, H., Burian, M. S., Michalik, S., Depke, M., Hildebrandt, P., Dhople, V. M., et al. (2014). Activation of the alternative sigma factor SigB of Staphylococcus aureus following internalization by epithelial cells-an in vivo proteomics perspective. Int. J. Med. Microbiol. 304, 177-187. doi: 10.1016/j.ijmm.2013. 11.014

Pförtner, H., Wagner, J., Surmann, K., Hildebrandt, P., Ernst, S., Bernhardt, J., et al. (2013). A proteomics workflow for quantitative and time-resolved analysis of adaptation reactions of internalized bacteria. Methods 61, 244-250. doi: 10.1016/j.ymeth.2013.04.009

Schilling, B., Rardin, M. J., Maclean, B. X., Zawadzka, A. M., Frewen, B. E., Cusack, M. P., et al. (2012). Platform-independent and label-free quantitation of proteomic data using MS1 extracted ion chromatograms in skyline: application to protein acetylation and phosphorylation. Mol. Cell. Proteomics 11, 202-214. doi: 10.1074/mcp.M112.017707

Schirle, M., Heurtier, M. A., and Kuster, B. (2003). Profiling core proteomes of human cell lines by one-dimensional PAGE and liquid chromatographytandem mass spectrometry. Mol. Cell. Proteomics 2, 1297-1305. doi: 10.1074/mcp.M300087-MCP200

Schmidt, F., Scharf, S. S., Hildebrandt, P., Burian, M., Bernhardt, J., Dhople, V., et al. (2010). Time-resolved quantitative proteome profiling of hostpathogen interactions: the response of Staphylococcus aureus RN1HG to internalisation by human airway epithelial cells. Proteomics 10, 2801-2811. doi: 10.1002/pmic.201000045

Shteynberg, D., Deutsch, E. W., Lam, H., Eng, J. K., Sun, Z., Tasman, N., et al. (2011). iProphet: multi-level integrative analysis of shotgun proteomic data improves peptide and protein identification rates and error estimates. Mol. Cell. Proteomics 10, M111.007690. doi: 10.1074/mcp.M111.007690

Sinha, B., François, P. P., Nüsse, O., Foti, M., Hartford, O. M., Vaudaux, P., et al. (1999). Fibronectin-binding protein acts as Staphylococcus aureus invasin via fibronectin bridging to integrin alpha5beta1. Cell. Microbiol. 1, 101-117. doi: 10.1046/j.1462-5822.1999.00011.x

Strelkov, S., Von Elstermann, M., and Schomburg, D. (2004). Comprehensive analysis of metabolites in Corynebacterium glutamicum by gas chromatography/mass spectrometry. Biol. Chem. 385, 853-861. doi: 10.1515/BC. 2004.111

Tuchscherr, L., Medina, E., Hussain, M., Völker, W., Heitmann, V., Niemann, S., et al. (2011). Staphylococcus aureus phenotype switching: an effective bacterial strategy to escape host immune response and establish a chronic infection. EMBO Mol. Med. 3, 129-141. doi: 10.1002/emmm.2010 00115

Vizcaino, J. A., Deutsch, E. W., Wang, R., Csordas, A., Reisinger, F., Rios, D., et al. (2014). ProteomeXchange provides globally coordinated proteomics data submission and dissemination. Nat. Biotechnol. 32, 223-226. doi: 10.1038/ nbt.2839

Wang, J. H., Zhang, K., Wang, N., Qiu, X. M., Wang, Y. B., and He, P. (2013). Involvement of phosphatidylinositol 3-Kinase/Akt signaling pathway in $\beta 1$ integrin-mediated internalization of Staphylococcus aureus by alveolar epithelial cells. J. Microbiol. 51, 644-650. doi: 10.1007/s12275-0133040-x

Wertheim, H. F., Melles, D. C., Vos, M. C., Van Leeuwen, W., Van Belkum, A., Verbrugh, H. A., et al. (2005). The role of nasal carriage in Staphylococcus aureus infections. Lancet Infect. Dis. 5, 751-762. doi: 10.1016/S1473-3099(05) 70295-4

Zeitlin, P. L., Lu, L., Rhim, J., Cutting, G., Stetten, G., Kieffer, K. A., et al. (1991). A cystic fibrosis bronchial epithelial cell line: immortalization by adeno-12SV40 infection. Am. J. Respir. Cell Mol. Biol. 4, 313-319. doi: 10.1165/ajrcmb/ 4.4 .313 
Conflict of Interest Statement: The authors declare that the research was conducted in the absence of any commercial or financial relationships that could be construed as a potential conflict of interest.

Received: 28 April 2014; accepted: 13 July 2014; published online: 01 August 2014. Citation: Surmann K, Michalik S, Hildebrandt P, Gierok P, Depke M, Brinkmann L, Bernhardt J, Salazar MG, Sun Z, Shteynberg D, Kusebauch U, Moritz RL, Wollscheid $B$, Lalk M, Völker U and Schmidt F (2014) Comparative proteome analysis reveals conserved and specific adaptation patterns of Staphylococcus aureus after internalization by different types of human non-professional phagocytic host cells. Front. Microbiol. 5:392. doi: 10.3389/fmicb.2014.00392
This article was submitted to Systems Microbiology, a section of the journal Frontiers in Microbiology.

Copyright (c) 2014 Surmann, Michalik, Hildebrandt, Gierok, Depke, Brinkmann, Bernhardt, Salazar, Sun, Shteynberg, Kusebauch, Moritz, Wollscheid, Lalk, Völker and Schmidt. This is an open-access article distributed under the terms of the Creative Commons Attribution License (CC BY). The use, distribution or reproduction in other forums is permitted, provided the original author(s) or licensor are credited and that the original publication in this journal is cited, in accordance with accepted academic practice. No use, distribution or reproduction is permitted which does not comply with these terms. 